\title{
Molecular mechanisms of suppressor of fused in regulating the hedgehog signalling pathway (Review)
}

\author{
DENGLIANG HUANG ${ }^{1,2}$, YITING WANG ${ }^{1,2}$, JIABIN TANG $^{1}$ and SHIWEN LUO ${ }^{1,2}$ \\ ${ }^{1}$ Center for Experimental Medicine; ${ }^{2}$ Jiangxi Key Laboratory of Molecular Diagnostics and Precision Medicine, \\ The First Affiliated Hospital of Nanchang University, Nanchang, Jiangxi 330006, P.R. China
}

Received June 25, 2017; Accepted October 17, 2017

DOI: $10.3892 / \mathrm{ol} .2018 .8142$

\begin{abstract}
Highly conserved throughout evolution, the hedgehog $(\mathrm{Hh})$ signalling pathway has been demonstrated to be involved in embryonic development, stem cell maintenance and tissue homeostasis in animals ranging from invertebrates to vertebrates. In the human body, a variety of cancer types are associated with the aberrantly activated $\mathrm{Hh}$ signalling pathway. Multiple studies have revealed suppressor of fused (Sufu) as a key negative regulator of this signalling pathway. In vertebrates, Sufu primarily functions as a tumor suppressor factor by interacting with and inhibiting glioma-associated oncogene homologues (GLIs), which are the terminal transcription factors of the Hh signalling pathway and belong to the Kruppel family of zinc finger proteins; by contrast, the regulation of Sufu itself remains relatively unclear. In the present review article, we focus on the effects of Sufu on the Hh signalling pathway in tumourigenesis and the molecular mechanisms underlying the regulation of GLI by Sufu. In addition, the factors modulating the activity of Sufu at post-transcriptional levels are also discussed.
\end{abstract}

\section{Contents}

1. Introduction

2. General characterization of Sufu

3. Sufu mutation and cancer predisposition

4. Mechanisms underlying the regulation of GLI by Sufu

5. Regulation of Sufu at the mRNA and protein level

6. Conclusions

Correspondence to: Dr Shiwen Luo, Center for Experimental Medicine, The First Affiliated Hospital of Nanchang University, 17 Yongwai Street, Nanchang, Jiangxi 330006, P.R. China E-mail: shiwenluo@ncu.edu.cn

Key words: hedgehog signalling, glioma-associated oncogene homologue transcription factor, suppressor of fused, human cancers, molecular mechanism

\section{Introduction}

Being a part of the system that specifies pattern formation during animal embryogenesis, hedgehog (Hh) signalling was initially discovered to regulate cuticle patterns in Drosophila more than 30 years ago (1); its existence in mammals suggested the strictly conservative roles of the Hh pathway in animal embryogenesis during evolution (2-4). Considering that development and tumourigenesis are essentially inextricable processes and that tumourigenesis may be the product of abnormal development under undesired circumstances, it is quite reasonable that deregulated $\mathrm{Hh}$ signalling was discovered as the promoter of human cancers. It was initially involved in basal cell nevus syndrome in 1996 (5,6). Subsequently, aberrantly active $\mathrm{Hh}$ pathways are closely linked to various human cancers (7), including ovarian (8), gastric (9), hepatocellular (10) and bladder cancers $(11,12)$, indicating its significant roles in adult tissue homeostasis maintenance. Therefore, Hh pathway-targeted inhibitors have been developed for cancer therapy (13). Though highly conserved, the Hh pathway is much more complex in vertebrates than in Drosophila, likely due to adaptations for the functional complexity of higher multicellular organisms throughout evolution. For instance, the mammalian counterpart of the Drosophila Hh ligand is the Hedgehog family of secreted proteins, which contains three members: Sonic Hedgehog (Shh), Indian Hedgehog (Ihh), and Desert Hedgehog (Dhh); these proteins perform overlapping but quite distinctive functions. Similarly, the function of the terminal transcription factor Cubitus interruptus (Ci) in Drosophila is assigned to three glioma-associated factors (GLI)-GLI1, GLI2 and GLI3-in mammals (14). In addition, the protein suppressor of fused (Sufu), a negative regulator of Hh signalling pathway, seems to display a more important function in vertebrates, where Sufu deletion led to embryo lethality in mice (15) but no visible phenotypic alterations in Drosophila (16).

Among the three $\mathrm{Hh}$ signalling pathways, the most intensively studied at present is the Shh pathway, which is generally delineated as follows. The Hh ligand binding to the twelve-span transmembrane protein Ptc1 (Patched1), relieves the suppressive effect of Ptcl on another seven-span transmembrane protein, smoothened (Smo). Active Smo transduces Hh signalling across the cytomembrane to activate GLI through incompletely clarified mechanisms that likely involve 
dissociating GLI from a suppressive complex containing Sufu. Finally, activated GLI enters the nucleus to transcriptionally activate a specific set of genes that contribute to certain cellular activities. Furthermore, alternative Hh signalling cascades, through which Hh signalling is transduced through Ptc1 and Smo but are not mediated by GLI, were identified to regulate pain perception (17) and cellular metabolism (18). These alternative cascades comprise non-canonical Hh signalling pathways, which will not be described herein (19). This review focuses within the realm of the canonical mammalian Shh signalling pathway, which will be simply described as the Hh pathway in the following sections.

\section{General characterization of Sufu}

In early studies $(1,16,20)$, Hh, fused ( $f u)$, Sufu, Ptch and Smo genes, which we now know are core components of the $\mathrm{Hh}$ signalling pathway, were determined to be involved in segment polarity establishment during the embryonic development of Drosophila. The Sufu gene, located in the 87C8 region of the third chromosome in Drosophila (16), encodes a 53-kDa protein with no significant homologies with known proteins (21); its inactivation did not display a visible phenotype but was capable of rescuing the 'fused' phenotype caused by the loss-of-function mutation of the fused gene (16). Thus, the gene was called suppressor of fused $(S u f u)$, and through interactions with $\mathrm{Fu}$ and $\mathrm{Ci}$, the Sufu protein suppresses Ci activity to negatively regulate the Drosophila Hh signalling pathway (22).

Subsequently, a series of studies identified Sufu in vertebrates (23), and its roles as a negative regulator were supported. For instance, Sufu in chicken and mice shows relatively high similarity at the amino acid level to Drosophila Sufu (24), and analyses revealed that Sufu, primarily expressed in tissues patterned by Hh signalling, interacts with and suppresses GLI isoforms to function as a negative regulator of mammalian $\mathrm{Hh}$ signalling $(25,26)$. Furthermore, some remarkable discoveries were made regarding the primary structure, tissue distribution, and biochemical and functional characteristics of human Sufu (27). i) Two isoforms of Sufu resulting from alternative splicing of primary mRNA were discovered, with the shorter isoform being truncated at the carboxy terminus; however, the difference in function between the two splicingisoforms remains to be clarified. ii) Through facilitating the interaction of ubiquitin-ligase Slimb/ß-TrCP with GLI (GLI1, GLI2 and GLI3), human Sufu could regulate the stability and subcellular localization of GLI, ultimately modulating Hh pathway activity. iii) Frequent deletion of the chromosomal region 10q24-q25 spanning the Sufu locus in glioblastoma, prostate cancer and other cancers suggests a tumour-suppressive role of Sufu $(28,29)$. In addition, a structural study of Sufu, which is conductive to illuminating its physical interactions with other proteins such as GLI, defined the crystal structure of the whole Sufu protein. In both human and Drosophila, the Sufu protein presents as a monomer consisting of two globular domains with a short linker between them $(30,31)$.

As described above, Sufu acts as a negative regulator of the Hh signalling pathway and plays essential roles in both embryogenesis and adult tissue homeostasis in species ranging from invertebrates to vertebrates. Loss-of-function of Sufu is sufficient to activate the Hh signalling pathway irrespective of the presence of the Hh ligand (32). In addition, the embryonic lethality in mice due to Sufu deletion (15) implies important functions of Sufu in mammals. These findings strongly suggest a central role of Sufu in the mammalian Hh pathway, which, along with our observation of loss of Sufu in colorectal cancer specimens, intrigued us greatly.

\section{Sufu mutation and cancer predisposition}

After the Hh pathway was implicated in human cancers by Ptc1 inactivation associated with basal cell carcinomas $(5,6)$, substantial studies have been conducted to explore the tumour-promoting effects and the underlying mechanisms of a hyperactive Hh pathway. Presently, multiple Sufu mutations have ben identified (shown in Table I), which are named according to the standard nomenclature recommendations of the HGVS (http://www.HGVS.org/mutnomen/) (33). These mutations determine abnormal forms of the Sufu protein among various human cancers, with the medulloblastoma of desmoplastic/nodular subtype being most abundantly studied, which is one of the five histologic subtypes of medulloblastoma and is characteristic of aberrant Hh pathway (34).

In both familial and sporadic desmoplastic medulloblastoma, multiple somatic and germline Sufu mutations that are likely to predispose individuals to tumourigenesis were identified in a series of studies (35-39), with some mutations, such as c.71del and c.71dul, showing incomplete penetrance of the predisposition to medulloblastoma for unknown reasons (40). Brugières et al reported that germline Sufu mutations are responsible for over $50 \%$ of seemingly sporadic desmoplastic/nodular medulloblastoma cases, especially in patients diagnosed at younger than 3 years of age (35). Although abnormal protein products speculated from the mutated $S u f u$ genes are thought to lose their capacity to suppress GLI, only a few of them have been studied with respect to their functional consequences. Studies taking advantage of genetically modified mice were performed to confirm the tumour-stimulating effects of the loss of Sufu. While $\mathrm{Sufu}^{-/}$mice present embryonic lethality with cephalic and neural tube defects, indicating a pivotal role of Sufu in mouse embryonic development, $\mathrm{Sufu}^{+/-}$mice displayed characteristic features of human Gorlin syndrome due to enhanced $\mathrm{Hh}$ signalling but were less prone to harbour tumour medulloblastoma and rhabdomyosarcoma compared with $\mathrm{ptch}^{+/}$individuals $(15,41)$. However, the absence of the p53 gene could facilitate tumourigenesis in $\mathrm{Sufu}^{+-}$mice. It was shown that $\mathrm{Sufu}^{+/} \mathrm{p} 53^{-/-}$mice but not $\mathrm{p} 53^{-/-}$individuals frequently developed medulloblastoma and rhabdomyosarcoma similar to those observed in $\mathrm{Ptcl}^{+/-}$mice $(32,42)$, suggesting that abrogation of Sufu activity remains a critical step to initiate tumours in the context of p53 inactivation. To some extent, this finding is in agreement with the "two-hit" theory of tumor initiation. The observation that $\mathrm{Sufu}^{+/-}$mice are less prone than $\mathrm{Ptcl}^{+/ /}$individuals to develop tumours may be due not only to the differences in functions of Sufu and Ptcl but also to the genetic background of the experimental mouse model. Taken together, these studies indicate a stimulatory role of Sufu inactivation in medulloblastoma. However, in sharp contrast to the observations of Sufu mutations in the studies above, no somatic or germ line mutations or altered expression in Sufu was revealed in a screen of 145 primitive neuroectodermal 
Table I. Germline Sufu mutations associated with predisposition mainly to desmoplastic medulloblastoma, not including non-pathogenic Sufu variants.

\begin{tabular}{|c|c|c|c|c|}
\hline Author, year & Location & Nucleotide change & Speculated protein change & (Refs.) \\
\hline Spurrell et al, 2016 & Exon 1 & c.71delC & p.Pro24ArgfsX72 & $(46)$ \\
\hline Spurrell et al, 2016 & Exon 1 & c.71delC & p.Ala25GlyfsX23 & $(46)$ \\
\hline Svärd et al, 2009; Koch et al, 2004 & Exon 1 & c.143insA & p.Gln48ProfsX25 & $(42,43)$ \\
\hline Koch et al, 2004 & Exon 1 & c. $44 \mathrm{C}>\mathrm{T}^{\mathrm{a}}$ & p.Pro15Leu & (43) \\
\hline Heby-Henricson et al, 2012 & Intron $1^{\mathrm{b}}$ & c. $182+3 \mathrm{~A}>\mathrm{T}$ & p.? & $(41)$ \\
\hline Svärd et al, 2009; Koch et al, 2004 & Intron $1^{\mathrm{b}}$ & c. $183-2 \mathrm{~A}>\mathrm{T}$ & p.? & $(42,43)$ \\
\hline Heby-Henricson et al, 2012 & Exon 2 & c.294_295dupCT & p.Tyr99SerfsX23 & $(41)$ \\
\hline Heby-Henricson et al, 2012 & Intron $2^{\mathrm{b}}$ & c.318-10delT & p.? & $(41)$ \\
\hline Heby-Henricson et al, 2012 & Exon 3 & c.318-?_454+?dup & p.Glu106-?_Glu152+?dup & $(41)$ \\
\hline Heby-Henricson et al, 2012 & Exon 3 & c. $422 \mathrm{~T}>\mathrm{G}$ & p.Met141Arg & $(41)$ \\
\hline Scott et al, 2006 & Exon 3 & c. $544 \mathrm{G}>\mathrm{T}$ & p.Asp182Tyr & (44) \\
\hline Scott et al, 2006 & Exon 3 & c. $550 \mathrm{C}>\mathrm{T}$ & p.Gln $184 X$ & (44) \\
\hline Goetz and Anderson, 2010 & Intron $6^{\mathrm{b}}$ & c. $756+1 \mathrm{G}>\mathrm{A}$ & p.? & (59) \\
\hline Zabidi and Stark, 2016 & Exon 7 & c.846insC & p.Glu283ArgfsX3 & $(45)$ \\
\hline Koch et al, 2004 & Exon 7 & c. $1018 \mathrm{G}>\mathrm{T}$ & p.Ala340Ser & (43) \\
\hline $\begin{array}{l}\text { Koch et al, 2004; Zabidi and Stark, 2016; } \\
\text { Endoh-Yamagami et al, } 2009\end{array}$ & Intron $8^{\mathrm{b}}$ & c. $1022+1 \mathrm{G}>\mathrm{A}^{\mathrm{a}}$ & p.? & $(43,45,61)$ \\
\hline Heby-Henricson et al, 2012 & Exon 9 & c. $1123 \mathrm{C}>\mathrm{T}$ & p.Gln $375 X$ & $(41)$ \\
\hline Heby-Henricson et al, 2012 & Exon 9 & c.1149_1150dupCT & p.Cys484ProfsX14 & $(41)$ \\
\hline Koch et al, 2004 & Exon 9 & c.1129_1135delTCCGGAG ${ }^{\mathrm{a}}$ & p.Ser377ProfsX7 & $(43)$ \\
\hline Heby-Henricson et al, 2012 & Intron $10^{\mathrm{b}}$ & c. $1297-1 \mathrm{G}>\mathrm{C}$ & p.? & (41) \\
\hline
\end{tabular}

${ }^{a}$ Mutations identified in tumours. ${ }^{b}$ Mutations in introns mainly occur at splice sites, likely leading to large fragment alterations. The nucleotides are numbered and the mutations are named according to the standard nomenclature recommendations of the HGVS (http://www.HGVS. org/mutnomen/). Sufu, suppressor of fused.

tumours, suggesting that genetic alteration of Sufu is a relatively rare event in human medulloblastoma (43). In addition, in a study to discover critical mutational targets in region 10q23.3-10q25.3, which contains Sufu and displays deletion in medulloblastoma, no tumour-specific sequence variations or DNA hypermethylation of promoter $\mathrm{CpG}$ islands of $S u f u$ and other known genes in that region were discovered (44). One explanation for these observed disparities in Sufu mutations among medulloblastoma tumours includes the heterogeneous characteristics of tumours. Moreover, it is also possible that in cancers harbouring hyperactive Hh pathways with intact Sufu loci, the function of Sufu is debilitated through epigenetic modifications of some unidentified regulatory sequences or translational and post-translational modifications of the Sufu protein. Nonetheless, these modifications cannot be detected with techniques involving the screening of only genetic mutations or by simply analysing putative regulatory genomic sequence $(45,46)$ or protein expression levels.

In addition to medulloblastoma, Sufu mutations have also been found in other human cancers. Applying genome-wide linkage analysis and exome sequencing, Aavikko et al revealed a germline Sufu mutation (c.367C $>\mathrm{T}$ ) in meningiomas encoding the protein mutant p.Arg123Cys. As a result, an altered tertiary structure and compromised function of Sufu occurred (47). Genetic mutations in Sufu and other Hh pathway components were also detected in mesothelioma (48) and rhabdomyoma (49). Some other studies have linked Sufu mutations to basal cell carcinoma (BCC) (50-52) and skin hamartomata (53). It was also reported that the Hh pathway hyperactivation attributed to the loss of Sufu or that overexpression of the Hh ligand may participate in the progression and metastases of prostate cancer (54), while the germline splicing mutation c. $1022+1 \mathrm{G}>\mathrm{A}$ generated a Sufu protein with a deletion of exon 8 instead of the general Ptcl mutation that was found in a family with atypical Gorlin syndrome (55).

In summary, consistent with the fact that Sufu is a critical suppressor of the Hh pathway, which promotes tumourigenesis when aberrantly activated, Sufu inactivation has been implicated in various human cancers. Subsequently, the molecular mechanisms underlying the regulation of $\mathrm{Hh}$ signalling activity by Sufu will be illustrated in detail.

\section{Mechanisms underlying the regulation of GLI by Sufu}

Within the realm of the Hh signalling pathway, substantial research focusing on Sufu has revealed multiple mechanisms by 


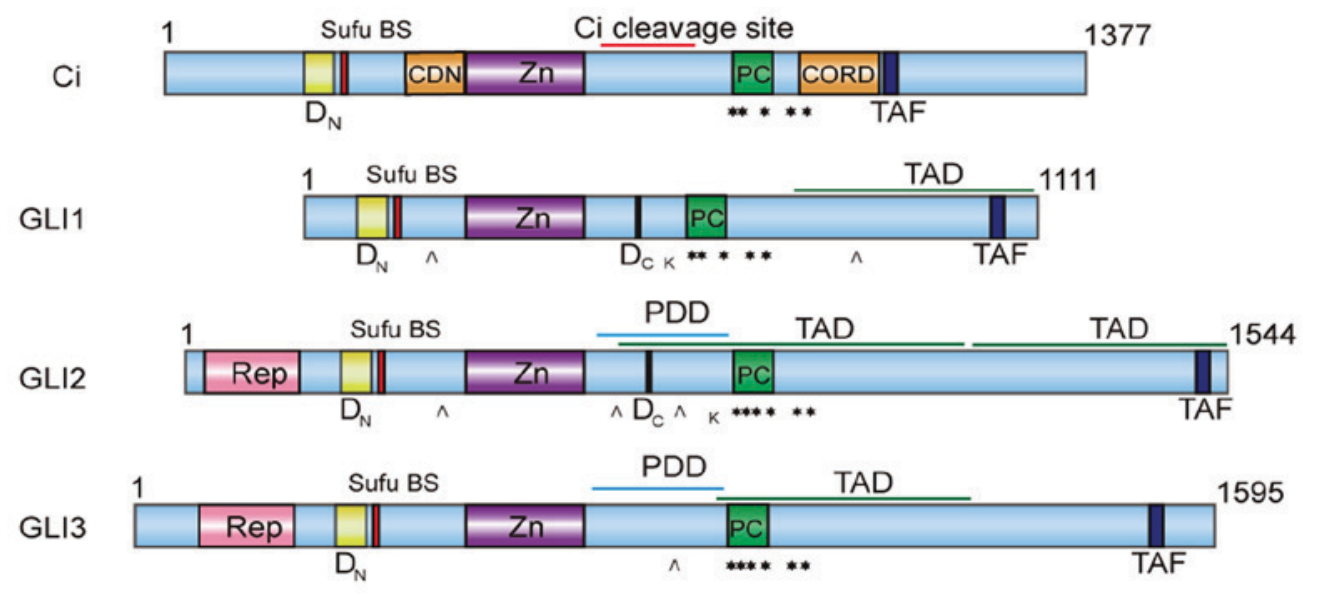

Figure 1. Schematic representation of domains, motifs and modification sites of Drosophila $\mathrm{Ci}$ and mouse GLI1, GLI2 and GLI3 (14). Ci and GLIs in the figure are aligned against the zinc-finger DNA-binding motif. In mammals, both GLI2 and GLI3 in full-length form can function as transcription activators, whereas under the influence of the processing determinant domain, GLI3 and GLI2, to a lesser extent, will be partially degraded by truncating their C-terminal TADs, resulting in transcription repression. In contrast, GLI1, lacking the processing determinant domain, and the N-terminal Reps in GLI2 and GLI3 can be activated by GLI2, resulting in constitutive transcription activation and indicating $\mathrm{Hh}$ pathway activity. In addition, the various motifs and modification sites may imply the high complexity of GLIs in terms of their function and regulation. Ci, Cubitus interruptus; GLI, glioma-associated oncogene homologue; Hh, hedgehog; Zn, zinc-finger DNA-binding domain; TAD, transcription activation domain; TAF, TAF-binding site acidic activation motif; Rep, repressor domain; CDN, CORD, Cos2-binding sites; Sufu BS, Sufu-binding site; PC, phosphorylation cluster; PDD, processing deteminant domain; $\mathrm{D}_{\mathrm{n}}$, $\mathrm{D}_{\mathrm{c}}$, degrons; "protein

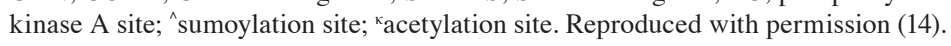

which it regulates the transcription activity of GLI, supporting its critical regulatory role in the Hh pathway. As a suppressor of the Hh pathway in both invertebrates and vertebrates, the regulation modes of GLI by Sufu in vertebrates are much more intricate than the regulation of Ci by Sufu in Drosophila (56), in part due to different regulation patterns of GLI1, GLI2 and GLI3 because of differences in structure (as shown in Fig. 1) and functionality among GLI proteins (14). As primary mediators of the Hh pathway, GLI2 mainly functions as a potential activator of the pathway, while GLI3 acts mainly as a suppressor $(57,58)$, although they both cooperate to modulate the expression of GLI1 and other target genes. Once generated, GLI1 functions as a constitutive activator to amplify $\mathrm{Hh}$ signalling due to the lack of an $\mathrm{N}$-terminal suppressor domain in GLI, which exists in GLI2 and GLI3 (14).

Hh signalling transduction is realized by alterations in the distributions of Hh pathway components, such as Ptc1, Smo, GLI and Sufu, within the primary cilium, a microtubule-based projection of the cell membrane that generally functions in vertebrates as a sensor of various extracellular signal pathways, including the Hh pathway (59). When the Hh signalling pathway is quiescent in the absence of functional Hh ligand, the binding of Sufu to GLI2 and GLI3 sequesters them in a cytoplasmic complex containing a cilium-associated kinesin Kif7 (60-62), known as the vertebrate homologue of Cos2 in Drosophila. This sequestration directly prohibits full-length GLI proteins from entering the nucleus and functioning as transcriptional activators. A study indicated that the binding of Sufu to GLI1 impeded the nuclear accumulation of GLI1 (63), consequently inhibiting proliferation, invasion and vascular mimicry of glioma cancer cells, while Sufu knockdown reversed these suppressive effects. Later, Sufu was reported to compete with importin $\beta 1$ to bind to the same site on GLI1, thus retarding the importin $\beta 1$-mediated nuclear translocation of GLI1 (64). Similarly, by masking the NLS (nuclear localization sequence), Sufu precludes Transportin-mediated nuclear import of $\mathrm{Ci}$ in Drosophila, as well as the nuclear accumulation of GLI2 and GLI3 mediated by Kapß2 (65), the mammalian homologue of Transportin. Recently, a study revealed that Sufu can sequester Ci/GLI proteins in the cytoplasm by binding to their $\mathrm{N}$-terminal regions, while in the nucleus, the suppression of $\mathrm{Ci} / \mathrm{GLI}$ activity is dependent on their C-terminal regions (66). Moreover, binding of Sufu to GLI2/3 in the cytoplasmic complex facilitates the production of repressor GLI3 (GLI3R) and, to a lesser extent, GLI2R, which result from the partial degradation of the full-length GLI2/3 and function as transcription suppressors (67). The hyper-phosphorylated full-length GLI2/3 resulting from sequential phosphorylation by protein kinase A (PKA), GSK3 $\beta$ and CK1 within the complex are partially degraded through the ubiquitin-proteasome pathway mediated by $S C^{\beta T r C P}$ ubiquitin E3 ligase, truncating the C-terminal transcriptional activator domain of GLI2/3 proteins $(68,69)$. In Drosophila, a cytoplasmic complex composed of fu, Sufu, Cos 2 and Ci similarly regulates $\mathrm{Ci}$ activity, suggesting some conserved scenario of Hh pathway regulation by Sufu during evolution. GLI2R and GLI3R likely compete only with full-length GLI2/3 for the same regulatory sequences to inhibit the otherwise activated transcription or may cooperate with co-repressors recruited in a GLI2/3R-dependent manner. Nevertheless, the inhibitory cytoplasmic complex described above would be transformed by Hh signalling $(70,71)$. Following Smo activation, the cytoplasmic complex containing Sufu and GLI2/3 is recruited to the primary cilium tip, where dissociation of the complex releases full-length GLI2 and GLI3 (72), which are subsequently translocated into the nucleus as potential transcriptional activators to take the place of GLIR, while Sufu is induced to degrade $(73,74)$. Recently, it was reported that responding to Hh signalling, Sufu accompanies GLI1 to the primary cilium, which is coupled to the subsequent nuclear transport of GLI1 (75). The necessity of the primary cilium for GLI derepression is supported by the report of a novel 
central domain characterized in GLI2 that is essential for both primary cilium localization and transcriptional activity (76). However, the specific mechanisms by which Hh signal is relayed from activated Smo in primary cilium to finally derepress GLI2/3 are far from fully understood. Recently, a primary cilium G protein-coupled receptor, Gpr161 (77), which also regulates the Wnt signalling pathway, was linked to Hh signalling transduction via Smo in primary cilium (78). Several studies have determined that the EVC/EVC2 ciliary complex, whose loss-of-function mutations contribute to Ellis-van Creveld syndrome, interacts with Smo to particularly regulate Sufu/GLI3 dissociation in primary cilium for normal endochondral bone formation, where Evc is needed for Hh pathway activation even when Sufu is deleted (79-81). In contrast, PKA is reported to efficiently suppress ciliary localization of GLI2-Sufu and GLI3-Sufu, while just moderately affecting GLI1-Sufu (72). While essential for antagonizing Sufu activity, the primary cilium is not required for the inhibitory effect of Sufu on the Hh pathway (82), and localization of Sufu in primary cilium is dependent on GLI, which alone is capable of accumulating in primary cilium (83).

However, considering the potently suppressive effect of Sufu on GLI activity, the finding that the protein levels of GLI2/3 in mice and Ci in Drosophila were reduced when Sufu was deleted seems paradoxical $(82,84)$. Sufu could retard proteasome-dependent degradation of GLI2/GLI3 promoted by Spop, a substrate-binding subunit for Cul3-based E3 ubiquitin ligase, by competing with Spop in binding the $\mathrm{N}$ - and $\mathrm{C}$-terminal regions of GLI3 and the C-terminal region of GLI2 (67). Notably, the stabilities of the GLI2R, GLI3R and GLI1 proteins are not influenced in this manner. Nevertheless, a recent study reported that Sufu is also essential for maintaining high protein level and nuclear accumulation of GLI1 in response to Hh signalling, sustaining the proliferation of granule neuron precursor cells (75). Mechanistically, Sufu-mediated stabilization of full-length GLI2/3 is of great importance. Without Hh signalling, the binding of Sufu to GLI2/3 preserves a pool of full-length GLI2/3 by preventing complete degradation stimulated by Spop. It also facilitates generation of GLI3R and, to a lesser extent, GLI2R medi-

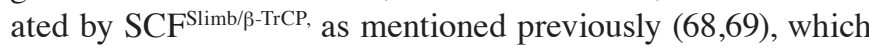
restrains gene transcription. As soon as $\mathrm{Hh}$ signalling is present, the preserved full-length GLI2/3 are derepressed to produce a transcriptional activator, which counteracts GLI2R and GLI3R, resulting in the rapid response of $\mathrm{Hh}$ signalling. Understandably, the activated full-length GLI $2 / 3$ are subjected to Spop-promoted degradation and are thus labile and unable to activate the Hh pathway constitutively, which is similar to the situation of Ci seen in Drosophila (85).

As in the cytoplasm, Sufu also regulates GLI proteins within the nucleus. For instance, it can recruit the SAP18-mSin3-HDAC co-repressor complex to suppress GLI transcriptional activity $(86,87)$. SAP18 is recruited to GLI3R-Sufu to form a suppressive complex occupying the GLI binding site when Hh signalling is quiescent, and upon Hh pathway activation this suppressive complex will be replaced by GLI1-Sufu (75) to activate the transcription of target genes. Other nuclear proteins mainly involved in basal transcription machinery were identified to interact with Sufu in a yeast two-hybrid screen using mouse Sufu as a bait, indicating crucial functions of Sufu in the nucleus. Sufu was revealed to interact with those other nuclear proteins and GLI proteins through the $\mathrm{N}$ - and C- termini, respectively, to form the suppressive complex (86). Similarly, p66 $\beta$ is the co-repressor that is recruited by Sufu to suppress GLI2/3 activity in the nucleus, whereas Hh signalling can induce Sufu/p66 $\beta$ dissociation from GLI and stimulate GLI activity (88). In addition, binding of Sufu in the nucleus also promotes GLI1 and GLI2 to export from the nucleus to the cytoplasm $(89,90)$. In C3H10T1/2 cells, treatment with leptomycin $\mathrm{B}$, which inhibits nuclear export by binding to the nuclear export receptor CRM1, dramatically shifts the localization of co-expressed GLI1/Sufu from the cytoplasm to the nucleus, suggesting that Sufu stimulates GLI1 export from the nucleus in a CRM1-dependent manner. However, CRM1 is not involved in Sufu-promoted export of GLI2 from the nucleus. It is possible that the inhibition of GLIs in the nucleus by Sufu is a remedy for its failure to sequester them in the cytoplasm.

The integrated regulations of GLI by Sufu are at least partially responsible for further modulating the Hh pathway to maintain graded Hh pathway activity $(14,91,92)$, which is primarily represented by the GLIA/GLIR ratio and is essential for realizing the complex physiological functions of the $\mathrm{Hh}$ pathway within multicellular organisms. For example, deletion of Sufu disrupts the gradient of Ptc1 expression, which displays a high-to-low gradient from ventral to dorsal areas of the wild-type mouse neural tube, similar to the Hh level gradient (67). Furthermore, Sufu deletion compromised Hh pathway activity in the ventral area of neural tube in $\mathrm{Sufu}^{-/}$mice because more full-length GLI2/3 proteins are completely degraded compared with the situation in wild-type mice; conversely, in the dorsal area of the neural tube, $\mathrm{Hh}$ pathway activity is enhanced because GLI3R expression is reduced due to the lack of full-length GLI3 in the absence of Sufu (67). Thus, in combination with other factors, such as

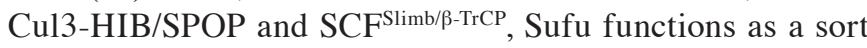
of a buffer for regulating GLI proteins (as shown in Fig. 2) that makes it possible to alter Hh pathway activity with high sensitivity in response to Hh signalling. Furthermore, Sufu is helpful for the interpretation of the Hh level gradient into graded Hh pathway activity.

Another important aspect of studies of GLI regulation by Sufu is the exploration of the protein structure of Sufu since it is helpful for explaining the physical interactions between Sufu and GLI (93), which is the basis for the regulations described above. As early as 2003 , it was reported that a 19-aa C-terminal fragment in Sufu was essential for the interaction with a novel motif SYGH within residues 116-125 in GLI1, which is consistent with the observation that among the three alternatively spliced transcripts of Sufu, two variants lacking the C-terminal fragment failed to interact with and suppress GLI1 (94). In addition, GLI1 with mutations in the SYGH motif is no longer suppressed by Sufu. Following arduous exploration, great progress has been made in the study of the Sufu protein crystal structure $(30,31)$, which in both humans and Drosophila presents as a monomer consisting of two globular domains-NTD (N-terminal domain) and CTD (C-terminal domain)-with a short linker in between. An 'open-to-closed' transition mode of Sufu conformation during interaction with GLI was proposed in both humans and Drosophila. In the analysis of the crystal structure of hSufu (with 286-345 aa 

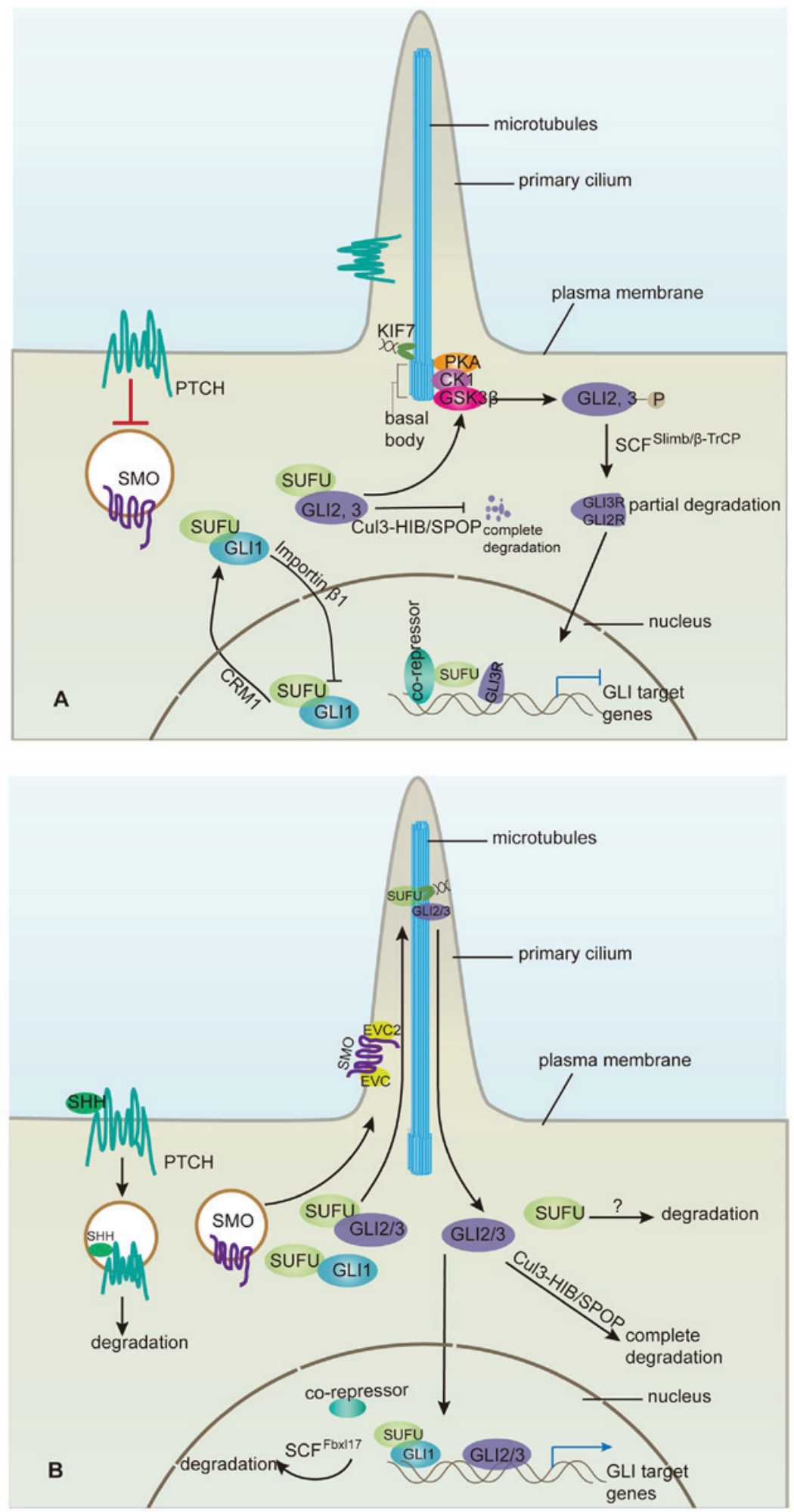

Figure 2. Regulation of GLI by Sufu in the Hh signalling pathway. (A) When the Hh pathway is quiescent without an Hh ligand, the GLI proteins are suppressed by Sufu through multiple mechanisms. In the cytoplasm, Sufu impedes importin $\beta 1$-mediated nuclear transport of GLI1, while nuclear Sufu promotes CRM1-mediated GLI1 export from the nucleus. In addition to impacting the nucleus-cytoplasm shuttle of GLI2 and GLI3, binding of Sufu to GLI2 and GLI3 facilitates the SCF (Slimb/ $\beta$-TrCP) ubiquitin ligase-mediated partial degradation of GLI2 and GLI3 following sequential phosphorylation by PKA, GSK3 $\beta$ and CK1. The partially degraded GLI2 and GLI3 are transported into the nucleus to function as transcription repressors (GLI2R, GLI3R), and Sufu can also recruit co-repressor, such as SAP18-mSin3-HDAC and p66ß to suppress GLI activity in the nucleus, which can be reversed by Hh signalling. Meanwhile, Sufu binding can preserve a pool of full-length GLI2 and GLI3 by blocking their Cul3-HIB/SPOP ubiquitin ligase-mediated complete degradation. (B) Following Smo activation by Hh ligand binding to Ptc1, the suppressive cytoplasmic complex containing Sufu, GLI2/3, Kif7 and other proteins is recruited to the primary cilium tip, where dissociation of the complex releases the preserved full-length GLI2 and GLI3, while Sufu is destabilized. Without suppression by Sufu, the full-length GLI2 and GLI3 can function as potential transcription activators; however, they are susceptible to complete Cul3-HIB/SPOP-promoted degradation. Thus, the transcription activators CLI2/3 are labile and cannot continuously activate gene transcription in the absence of Hh signalling, and CLI1 is also transported into the nucleus accompanied with Sufu to stabilize it. In the nucleus, destabilization of Sufu, such as SCF (Fbxl17) uibiquitin ligase-mediated degradation of Sufu, results in dissociation of the SAP18-mSin3-HDAC-containing suppressive complex. GLI, glioma-associated oncogene homologue; Sufu, suppressor of fused; Hh, hedgehog; Fbxl17, F-box and leucine-rich repeat protein 17; Smo, smoothened. 
deleted) in a complex with hGLI1 (112-128aa) containing the SYGHLS motif, Sufu bound to the GLI1 peptide via clamping to the NTD and the CTD, showing the closed conformation of Sufu. This complex could be induced by Hh signalling to dissociate, which most likely occurs by transformation of Sufu from a closed to an open conformation, while the specific mechanism remains to be defined. Moreover, the key residues mediating the association between GLI and Sufu were further investigated through the analysis of the crystal structure in combination with site-specific mutagenesis.

To recapitulate the findings above, depending on the physical interaction between Sufu and GLI in combination with other factors, Sufu is engaged in regulating GLI protein along with the signalling transduction from the cytoplasm into the nucleus, modulating both the subcellular localization and stability of GLI to exquisitely realize the graded activity of GLI proteins in response to the Hh signalling gradient. These sophisticated regulations of GLI by Sufu indicate a central position of GLI-Sufu correlation downstream of Smo in the canonical mammalian $\mathrm{Hh}$ signalling pathway.

\section{Regulation of Sufu at the mRNA and protein level}

In contrast to the relatively extensive accomplishments in the regulation of GLI by Sufu, how Sufu itself is regulated by other factors remains obscure, although there have been some achievements in the study of Sufu regulation at the post-transcriptional level. Given the critical suppressive role of Sufu in the $\mathrm{Hh}$ pathway, it is conceivable that Sufu deactivation should be one of the prerequisites of $\mathrm{Hh}$ pathway activation, as was previously reported. Hh signalling, especially Shh, is capable of destabilizing the Sufu protein through inducing Sufu polyubiquitination at K257 and its subsequent degradation by the proteasome. In contrast, a K257R mutant of Sufu is, to a large extent, resistant to Hh signalling-induced degradation and exhibits a more potent suppressive function against cell growth (73), suggesting that the much more reduced stability of the Sufu protein is likely responsible for cancer development when there is an excess of Hh ligand. Though ubiquitin-proteasome pathway-mediated degradation is of great importance for Sufu regulation at the post-translational level, the exact ubiquitin ligase involved in this process remains unknown. Further studies revealed that phosphorylation also plays an important role in regulating Sufu stability. When $\mathrm{Hh}$ binds to Ptc1, following recruitment and dissociation of the Sufu/GLI complex in primary cilium, GLIs are released to enter the nucleus, while Sufu was transported out of primary cilium and degraded via the proteasome-dependent pathway. Dual phosphorylation at Ser-346 and Ser-342 by PKA and GSK3 $\beta$ can prolong the stay of Sufu in primary cilium and stabilize the protein (95). Recently, a study showed that the E3 ubiquitin ligase Skp1-Cul1-F-box protein Fbxl17 (F-box and leucine-rich repeat protein 17) targets Sufu proteolysis in the nucleus and promotes medulloblastoma (74), thus bridging the above ubiquitination and phosphorylation modifications in Sufu protein regulation. In response to Hh signalling, phosphorylation at Ser-346 and Ser-342 by PKA and GSK3 $\beta$ is debilitated, while Sufu dephosphorylation facilitates its polyubiquitination at $\mathrm{K} 257$ by $\mathrm{SCF}^{\mathrm{Fbx} x 17}$ and subsequent degradation, leading to Hh pathway activation.

Given the crucial modulatory effects of Sufu on GLI in both the cytoplasm and the nucleus, and the existence of different ubiquitin ligases responsible for GLI ubiquitination, such as Cul3-HIB/SPOP and $\mathrm{SCF}^{\text {Slimb/3-TrCP }}$, other ubiquitin ligases for Sufu ubiquitination may exist in the cytoplasm that are distinct from $\mathrm{SCF}^{\mathrm{Fbx} 117}$, which ubiquitinates nuclear Sufu. Additionally, our team recently identified NIMA-related expressed kinase 2A (Nek2A) as a Sufu-interacting protein by a yeast two-hybrid screen; the enzyme could phosphorylate and stabilize Sufu, consequently dampening Hh/GLI2 signalling $(96,97)$. It is likely that different post-translational modifications, including phosphorylation and ubiquitination, are orchestrated to modulate the stability of the Sufu protein, although additional studies are required to unveil other regulations and the relationships between them.

In addition to regulating the stability of the Sufu protein, $\mathrm{Hh}$ signalling also attenuates Sufu activity through suppressing the maturation of Sufu mRNA. In Drosophila, the target gene HIB (Hh-induced protein) of the Hh pathway is a substrate recognition component of Cul3-based ubiquitin ligase. In response to $\mathrm{Hh}$ signalling, the ubiquitin ligase $\mathrm{Cul3}-\mathrm{HIB}$, through poorly clarified mechanisms, stimulates enrichment of the spliceosome factor crooked neck (Crn) in the nucleus, which likely inhibits the formation of functional sufu mRNA and ultimately weakens Sufu activity. Moreover, a similar mechanism may also exist in mammals. Spop, the mammalian homologue of HIB, is also capable of downregulating Sufu levels in Drosophila (98). Other factors discovered to impact Sufu mRNA include micro-RNAs. MicroRNA-378 can promote cell survival, tumour growth and angiogenesis through attenuating the translation of both Sufu and Fus-1 (99). Furthermore, microRNA-214 can directly target Sufu mRNA and compromise its function, which facilitates lung adenocarcinoma metastasis and the epithelial-mesenchymal transition (EMT), which are most likely attributed to deregulated Hh signalling (100). Shown here, microRNA-214 and microRNA-378 display tumour-promoting roles, whereas some studies reported tumour-suppressive roles in cervical and breast cancer cells $(101,102)$, implying the intrinsic complexity of multi-cellular organisms and the considerable influence of the microenvironment on gene functions.

Although the mechanism underlying Sufu regulation remains obscure, its exploration is mounting, as the various post-transcriptional regulations that have been identified have begun to delineate the regulatory system focusing on Sufu. These intricate regulatory mechanisms at different levels, through the overall process of gene expression and at the post-translational level, remain to be clarified.

\section{Conclusions}

While a substantial number of studies have examined the $\mathrm{Hh}$ signalling pathway, some mechanisms within the Hh signalling cascade remain elusive. For instance, i) how is Smo inhibited by Ptc1? ii) How does the relay of Hh signalling from activated Smo to GLI occur? iii) How is the specific transcription by GLI proteins realized? and iv) Given the pivotal role of Sufu in Hh signalling, the study on the regulation of Sufu itself seems somewhat inadequate, with no studies reporting on the transcription of the $S u f u$ gene. In addition, what has been presented in this review is merely part of the whole picture, especially since Sufu is also implicated in the regulation of the Wnt pathway $(103,104)$, 
probably coordinating the Wnt and Hh pathways for their proper functions. In addition, Sufu was first associated with human immunity by its single-nucleotide polymorphism involvement in graft-versus-host disease (105). Furthermore, in a study on non-canonical Hh signalling, Sufu binds to and stabilizes cellular nucleic acid-binding protein (CNBP) to promote polyamine biosynthesis, which supports neuronal and medulloblastoma cell growth (106), suggesting a tumour-stimulating effect of Sufu. This seeming contradiction is likely due to our general inclination to assign definite functions to certain proteins within linear-pattern cellular signalling pathways, which are in actuality more like networks with proteins functioning in context-dependent manners. Perhaps a panoramic view is needed to understand these interconnected networks.

\section{References}

1. Nüsslein-Volhard $\mathrm{C}$ and Wieschaus E: Mutations affecting segment number and polarity in Drosophila. Nature 287: 795-801, 1980 .

2. Briscoe $\mathrm{J}$ and Thérond PP: The mechanisms of Hedgehog signalling and its roles in development and disease. Nat Rev Mol Cell Biol 14: 416-429, 2013.

3. Ingham PW, Nakano Y and Seger C: Mechanisms and functions of Hedgehog signalling across the metazoa. Nat Rev Genet 12: 393-406, 2011.

4. Varjosalo $\mathrm{M}$ and Taipale J: Hedgehog: Functions and mechanisms. Genes Dev 22: 2454-2472, 2008.

5. Johnson RL, Rothman AL, Xie J, Goodrich LV, Bare JW, Bonifas JM, Quinn AG, Myers RM, Cox DR, Epstein EH Jr and Scott MP: Human homolog of patched, a candidate gene for the basal cell nevus syndrome. Science 272: 1668-1671, 1996.

6. Hahn H, Wicking C, Zaphiropoulous PG, Gailani MR, Shanley S Chidambaram A, Vorechovsky I, Holmberg E, Unden AB, Gillies S, et al: Mutations of the human homolog of Drosophila patched in the nevoid basal cell carcinoma syndrome. Cell 85: 841-851, 1996.

7. Barakat MT, Humke EW and Scott MP: Learning from Jekyll to control Hyde: Hedgehog signaling in development and cancer Trends Mol Med 16: 337-348, 2010.

8. Chen Q, Gao G and Luo S: Hedgehog signaling pathway and ovarian cancer. Chin J Cancer Res 25: 346-353, 2013.

9. Zeng C, Wang Y,Lu Q, Chen J,Zhang J, Liu T, Lv N and Luo S: SPOP suppresses tumorigenesis by regulating Hedgehog/Gli2 signaling pathway in gastric cancer. J Exp Clin Cancer Res 33: 75, 2014.

10. Shi C, Huang D, Lu N, Chen D, Zhang M, Yan Y, Deng L, Lu Q, Lu $\mathrm{H}$ and Luo S: Aberrantly activated Gli2-KIF20A axis is crucial for growth of hepatocellular carcinoma and predicts poor prognosis. Oncotarget 7: 26206-26219, 2016.

11. Shin K, Lim A, Zhao C, Sahoo D, Pan Y, Spiekerkoetter E, Liao JC and Beachy PA: Hedgehog signaling restrains bladder cancer progression by eliciting stromal production of urothelial differentiation factors. Cancer Cell 26: 521-533, 2014.

12. Fei DL, Sanchez-Mejias A, Wang Z, Flaveny C, Long J, Singh S Rodriguez-Blanco J, Tokhunts R, Giambelli C, Briegel KJ, et al: Hedgehog signaling regulates bladder cancer growth and tumorigenicity. Cancer Res 72: 4449-4458, 2012.

13. Rimkus TK, Carpenter RL, Qasem S, Chan M and Lo HW: Targeting the sonic hedgehog signaling pathway: Review of smoothened and GLI inhibitors. Cancers (Basel) 8: pii: E22, 2016

14. Hui CC and Angers S: Gli proteins in development and disease. Annu Rev Cell Dev Biol 27: 513-537, 2011.

15. Svärd J, Heby-Henricson K, Persson-Lek M, Rozell B, Lauth M, Bergström A, Ericson J, Toftgård R and Teglund S: Genetic elimination of suppressor of fused reveals an essential repressor function in the mammalian hedgehog signaling pathway. Dev Cell 10: 187-197, 2006.

16. Préat T: Characterization of suppressor of fused, a complete suppressor of the fused segment polarity gene of Drosophila melanogaster. Genetics 132: 725-736, 1992.

17. Babcock DT, Shi S, Jo J, Shaw M, Gutstein HB and Galko MJ: Hedgehog signaling regulates nociceptive sensitization. Curr Biol 21: 1525-1533, 2011 .
18. Teperino R, Amann S, Bayer M, McGee SL, Loipetzberger A, Connor T, Jaeger C, Kammerer B, Winter L, Wiche G, et al: Hedgehog partial agonism drives Warburg-like metabolism in muscle and brown fat. Cell 151: 414-426, 2012.

19. Teperino R, Aberger F, Esterbauer H, Riobo N and Pospisilik JA: Canonical and non-canonical Hedgehog signalling and the control of metabolism. Semin Cell Dev Biol 33: 81-92, 2014.

20. Hooper JE: Distinct pathways for autocrine and paracrine Wingless signalling in Drosophila embryos. Nature 372: 461-464, 1994.

21. Pham A,TherondP,Alves G,TournierFB,Busson D,Lamour-Isnard C, Bouchon BL, Préat T and Tricoire H: The suppressor of fused gene encodes a novel PEST protein involved in Drosophila segment polarity establishment. Genetics 140: 587-598, 1995.

22. Monnier V, Dussillol F, Alves G, Lamour-Isnard C and Plessis A: Suppressor of fused links fused and Cubitus interruptus on the hedgehog signalling pathway. Curr Biol 8: 583-586, 1998.

23. Delattre M, Briand S, Paces-Fessy M and Blanchet-Tournier MF: The Suppressor of fused gene, involved in Hedgehog signal transduction in Drosophila, is conserved in mammals. Dev Genes Evol 209: 294-300, 1999.

24. Pearse RV II, Collier LS, Scott MP and Tabin CJ: Vertebrate homologs of Drosophila suppressor of fused interact with the gli family of transcriptional regulators. Dev Biol 212: 323-336, 1999.

25. Ding Q, Fukami Si, Meng X, Nishizaki Y, Zhang X, Sasaki H, Dlugosz A, Nakafuku M and Hui Cc: Mouse suppressor of fused is a negative regulator of sonic hedgehog signaling and alters the subcellular distribution of Gli1. Curr Biol 9: 1119-1122, 1999.

26. Simon-Chazottes D, Paces-Fessy M, Lamour-Isnard C, Guénet JL and Blanchet-Tournier MF: Genomic organization, chromosomal assignment, and expression analysis of the mouse suppressor of fused gene (Sufu) coding a Gli protein partner. Mamm Genome 11: 614-621, 2000.

27. Stone DM, Murone M, Luoh S, Ye W, Armanini MP, Gurney A, Phillips H, Brush J, Goddard A, de Sauvage FJ and Rosenthal A: Characterization of the human suppressor of fused, a negative regulator of the zinc-finger transcription factor Gli. J Cell Sci 112: 4437-4448, 1999.

28. Rasheed BK, McLendon RE, Friedman HS, Friedman AH, Fuchs HE, Bigner DD and Bigner SH: Chromosome 10 deletion mapping in human gliomas: A common deletion region in 10q25. Oncogene 10: 2243-2246, 1995.

29. Gray IC, Phillips SM, Lee SJ, Neoptolemos JP, Weissenbach J and Spurr NK: Loss of the chromosomal region 10q23-25 in prostate cancer. Cancer Res 55: 4800-4803, 1995.

30. Cherry AL, Finta C, Karlström M, Jin Q, Schwend T, Astorga-Wells J, Zubarev RA, Del Campo M, Criswell AR, de Sanctis D, et al: Structural basis of SUFU-GLI interaction in human Hedgehog signalling regulation. Acta Crystallogr D Biol Crystallogr 69: 2563-2579, 2013.

31. Zhang Y, Fu L, Qi X, Zhang Z, Xia Y, Jia J, Jiang J, Zhao Y and Wu G: Structural insight into the mutual recognition and regulation between Suppressor of Fused and Gli/Ci. Nat Commun 4: 2608, 2013.

32. Lee Y, Kawagoe R, Sasai K, Li Y, Russell HR, Curran T and McKinnon PJ: Loss of suppressor-of-fused function promotes tumorigenesis. Oncogene 26: 6442-6447, 2007.

33. Ogino S, Gulley ML, den Dunnen JT and Wilson RB; Association for Molecular Patholpogy Training and Education Committee: Standard mutation nomenclature in molecular diagnostics: Practical and educational challenges. J Mol Diagn 9: 1-6, 2007.

34. Louis DN, Ohgaki H, Wiestler OD, Cavenee WK, Burger PC, Jouvet A, Scheithauer BW and Kleihues P: The 2007 WHO classification of tumours of the central nervous system. Acta Neuropathol 114: 97-109, 2007.

35. Brugières L, Remenieras A, Pierron G, Varlet P, Forget S, Byrde V, Bombled J, Puget S, Caron O, Dufour C, et al: High frequency of germline SUFU mutations in children with desmoplastic/nodular medulloblastoma younger than 3 years of age. J Clin Oncol 30: 2087-2093, 2012.

36. Ng D, Stavrou T, Liu L, Taylor MD, Gold B, Dean M, Kelley MJ, Dubovsky EC, Vezina G, Nicholson HS, et al: Retrospective family study of childhood medulloblastoma. Am J Med Genet A 134: 399-403, 2005.

37. Taylor MD, Liu L, Raffel C, Hui CC, Mainprize TG, Zhang X Agatep R, Chiappa S, Gao L, Lowrance A, et al: Mutations in SUFU predispose to medulloblastoma. Nat Genet 31: 306-310, 2002.

38. Smith MJ, Beetz C, Williams SG, Bhaskar SS, O'Sullivan J, Anderson B, Daly SB, Urquhart JE, Bholah Z, Oudit D, et al: Germline mutations in SUFU cause Gorlin syndrome-associated childhood medulloblastoma and redefine the risk associated with PTCH1 mutations. J Clin Oncol 32: 4155-4161, 2014. 
39. Slade I, Murray A, Hanks S, Kumar A, Walker L, Hargrave D, Douglas J, Stiller C, Izatt L and Rahman N: Heterogeneity of familial medulloblastoma and contribution of germline PTCH1 and SUFU mutations to sporadic medulloblastoma. Fam Cancer 10: 337-342, 2011.

40. Brugières L, Pierron G, Chompret A, Paillerets BB, Di Rocco F, Varlet P, Pierre-Kahn A, Caron O, Grill J and Delattre O: Incomplete penetrance of the predisposition to medulloblastoma associated with germ-line SUFU mutations. J Med Genet 47: $142-144,2010$.

41. Heby-Henricson K, Bergström A, Rozell B, Toftgård R and Teglund S: Loss of Trp53 promotes medulloblastoma development but not skin tumorigenesis in Sufu heterozygous mutant mice. Mol Carcinog 51: 754-760, 2012.

42. Svärd J, Rozell B, Toftgård R and Teglund S: Tumor suppressor gene co-operativity in compound Patched 1 and suppressor of fused heterozygous mutant mice. Mol Carcinog 48: 408-419, 2009.

43. Koch A, Waha A, Hartmann W, Milde U, Goodyer CG, Sörensen N, Berthold F, Digon-Söntgerath B, Krätzschmar J, Wiestler OD and Pietsch T: No evidence for mutations or altered expression of the Suppressor of Fused gene (SUFU) in primitive neuroectodermal tumours. Neuropathol Appl Neurobiol 30: 532-539, 2004

44. Scott DK, Straughton D, Cole M, Bailey S, Ellison DW and Clifford SC: Identification and analysis of tumor suppressor loci at chromosome 10q23.3-10q25.3 in medulloblastoma. Cell Cycle 5: 2381-2389, 2006

45. Zabidi MA and Stark A: Regulatory enhancer-core-promoter communication via transcription factors and cofactors. Trends Genet 32: 801-814, 2016.

46. Spurrell CH, Dickel DE and Visel A: The ties that bind: Mapping the dynamic enhancer-promoter interactome. Cell 167: 1163-1166, 2016

47. Aavikko M, Li SP, Saarinen S, Alhopuro P, Kaasinen E, Morgunova E, Li Y, Vesanen K, Smith MJ, Evans DG, et al: Loss of SUFU function in familial multiple meningioma. Am J Hum Genet 91: 520-526, 2012.

48. Lim CB, Prêle CM, Cheah HM, Cheng YY, Klebe S, Reid G Watkins DN, Baltic S, Thompson PJ and Mutsaers SE: Mutational analysis of hedgehog signaling pathway genes in human malignant mesothelioma. PLoS One 8: e66685, 2013.

49. Tostar U, Malm CJ, Meis-Kindblom JM, Kindblom LG, Toftgård R and Undén AB: Deregulation of the hedgehog signalling pathway: A possible role for the PTCH and SUFU genes in human rhabdomyoma and rhabdomyosarcoma development. J Pathol 208: 17-25, 2006

50. Yin WC, Li ZJ and Hui CC: BCC or not: Sufu keeps it in check. Oncoscience 2: 77-78, 2015.

51. Schulman JM, Oh DH, Sanborn JZ, Pincus L, McCalmont TH and Cho RJ: Multiple hereditary infundibulocystic basal cell carcinoma syndrome associated with a germline SUFU mutation. JAMA Dermatol 152: 323-327, 2016.

52. Reifenberger J, Wolter M, Knobbe CB, Köhler B, Schönicke A, Scharwächter C, Kumar K, Blaschke B, Ruzicka T and Reifenberger G: Somatic mutations in the PTCH, SMOH, SUFUH and TP53 genes in sporadic basal cell carcinomas. Br J Dermatol 152: 43-51, 2005

53. Mann K, Magee J, Guillaud-Bataille M, Blondel C, Bressac-de Paillerets B, Yeatman J and Winship I: Multiple skin hamartomata: A possible novel clinical presentation of SUFU neoplasia syndrome. Fam Cancer 14: 151-155, 2015.

54. Sheng T, Li C, Zhang X, Chi S, He N, Chen K, McCormick F, Gatalica $\mathrm{Z}$ and Xie J: Activation of the hedgehog pathway in advanced prostate cancer. Mol Cancer 3: 29, 2004

55. Pastorino L, Ghiorzo P, Nasti S, Battistuzzi L, Cusano R, Marzocchi C, Garrè ML, Clementi M and Scarrà GB: Identification of a SUFU germline mutation in a family with Gorlin syndrome. Am J Med Genet A 149A: 1539-1543, 2009.

56. Ruel L and Thérond PP: Variations in Hedgehog signaling: Divergence and perpetuation in Sufu regulation of Gli. Genes Dev 23: 1843-1848, 2009.

57. McDermott A, Gustafsson M, Elsam T, Hui CC, Emerson CP Jr and Borycki AG: Gli2 and Gli3 have redundant and context-dependent function in skeletal muscle formation. Development 132 : 345-357, 2005

58. Pan Y, Wang C and Wang B: Phosphorylation of Gli2 by protein kinase $\mathrm{A}$ is required for Gli2 processing and degradation and the Sonic Hedgehog-regulated mouse development. Dev Biol 326: 177-189, 2009
59. Goetz SC and Anderson KV: The primary cilium: A signalling centre during vertebrate development. Nat Rev Genet 11: 331-344, 2010.

60. Wen X, Lai CK, Evangelista M, Hongo JA, de Sauvage FJ and Scales SJ: Kinetics of hedgehog-dependent full-length Gli3 accumulation in primary cilia and subsequent degradation. Mol Cell Biol 30: 1910-1922, 2010

61. Endoh-Yamagami S, Evangelista M, Wilson D, Wen X, Theunissen JW, Phamluong K, Davis M, Scales SJ, Solloway MJ, de Sauvage FJ and Peterson AS: The mammalian Cos2 homolog Kif7 plays an essential role in modulating $\mathrm{Hh}$ signal transduction during development. Curr Biol 19: 1320-1326, 2009.

62. Cheung HO, Zhang X, Ribeiro A, Mo R, Makino S, Puviindran V, Law KK, Briscoe J and Hui CC: The kinesin protein Kif7 is a critical regulator of Gli transcription factors in mammalian hedgehog signaling. Sci Signal 2: ra29, 2009.

63. Liu X, Wang X, Du W, Chen L, Wang G, Cui Y, Liu Y, Dou Z, Wang H, Zhang P, et al: Suppressor of fused (Sufu) represses Gli1 transcription and nuclear accumulation, inhibits glioma cell proliferation, invasion and vasculogenic mimicry, improving glioma chemo-sensitivity and prognosis. Oncotarget 5: 11681-11694, 2014.

64. Szczepny A, Wagstaff KM, Dias M, Gajewska K, Wang C, Davies RG, Kaur G, Ly-Huynh J, Loveland KL and Jans DA: Overlapping binding sites for importin b1 and suppressor of fused $(\mathrm{SuFu})$ on glioma-associated oncogene homologue 1 (Gli1) regulate its nuclear localization. Biochem J 461: 469-476, 2014.

65. Shi Q, Han Y and Jiang J: Suppressor of fused impedes Ci/Gli nuclear import by opposing Trn/Kapb2 in Hedgehog signaling. J Cell Sci 127: 1092-1103, 2014.

66. Han Y, Shi Q and Jiang J: Multisite interaction with Sufu regulates $\mathrm{Ci} / \mathrm{Gli}$ activity through distinct mechanisms in $\mathrm{Hh}$ signal transduction. Proc Natl Acad Sci USA 112: 6383-6388, 2015.

67. Wang C, Pan Y and Wang B: Suppressor of fused and Spop regulate the stability, processing and function of Gli2 and Gli3 full-length activators but not their repressors. Development 137: 2001-2009, 2010.

68. Tempé D, Casas M, Karaz S, Blanchet-Tournier MF and Concordet JP: Multisite protein kinase A and glycogen synthase kinase 3beta phosphorylation leads to Gli3 ubiquitination by SCFbetaTrCP. Mol Cell Biol 26: 4316-4326, 2006.

69. Wang B and Li Y: Evidence for the direct involvement of $\{$ beta\} TrCP in Gli3 protein processing. Proc Natl Acad Sci USA 103: 33-38, 2006.

70. Pan Y, Bai CB, Joyner AL and Wang B: Sonic hedgehog signaling regulates Gli2 transcriptional activity by suppressing its processing and degradation. Mol Cell Biol 26: 3365-3377, 2006.

71. Wang B, Fallon JF and Beachy PA: Hedgehog-regulated processing of Gli3 produces an anterior/posterior repressor gradient in the developing vertebrate limb. Cell 100: 423-434, 2000.

72. Tukachinsky H, Lopez LV and Salic A: A mechanism for vertebrate Hedgehog signaling: Recruitment to cilia and dissociation of SuFu-Gli protein complexes. J Cell Biol 191: 415-428, 2010.

73. Yue S, Chen Y and Cheng SY: Hedgehog signaling promotes the degradation of tumor suppressor Sufu through the ubiquitin-proteasome pathway. Oncogene 28: 492-499, 2009.

74. Raducu M, Fung E, Serres S, Infante P, Barberis A, Fischer R, Bristow C, Thézénas ML, Finta C, Christianson JC, et al: SCF (Fbxl17) ubiquitylation of Sufu regulates Hedgehog signaling and medulloblastoma development. EMBO J 35: 1400-1416, 2016.

75. Zhang Z, Shen L, Law K, Zhang Z, Liu X, Hua H, Li S, Huang H, Yue S, Hui CC and Cheng SY: Suppressor of fused chaperones Gli proteins to generate transcriptional responses to sonic hedgehog signaling. Mol Cell Biol 37: pii: e00421-16, 2017.

76. Santos N and Reiter JF: A central region of Gli2 regulates its localization to the primary cilium and transcriptional activity. J Cell Sci 127: 1500-1510, 2014.

77. Li BI, Matteson PG, Ababon MF, Nato AQ Jr, Lin Y, Nanda V, Matise TC and Millonig JH: The orphan GPCR, Gpr161, regulates the retinoic acid and canonical Wnt pathways during neurulation. Dev Biol 402: 17-31, 2015.

78. Pal K, Hwang SH, Somatilaka B, Badgandi H, Jackson PK, DeFea K and Mukhopadhyay S: Smoothened determines b-arrestin-mediated removal of the $\mathrm{G}$ protein-coupled receptor Gpr161 from the primary cilium. J Cell Biol 212: 861-875, 2016.

79. Blair HJ, Tompson S, Liu YN, Campbell J, MacArthur K, Ponting CP, Ruiz-Perez VL and Goodship JA: Evc2 is a positive modulator of Hedgehog signalling that interacts with Evc at the cilia membrane and is also found in the nucleus. BMC Biol 9: 14, 2011. 
80. Yang C, Chen W, Chen Y and Jiang J: Smoothened transduces Hedgehog signal by forming a complex with Evc/Evc2. Cell Res 22: 1593-1604, 2012.

81. Caparrós-Martín JA, Valencia M, Reytor E, Pacheco M, Fernandez M, Perez-Aytes A, Gean E, Lapunzina P, Peters H, Goodship JA and Ruiz-Perez VL: The ciliary Evc/Evc2 complex interacts with Smo and controls Hedgehog pathway activity in chondrocytes by regulating Sufu/Gli3 dissociation and Gli3 trafficking in primary cilia. Hum Mol Genet 22: 124-139, 2013.

82. Jia J, Kolterud A, Zeng H, Hoover A, Teglund S, Toftgård R and Liu A: Suppressor of Fused inhibits mammalian Hedgehog signaling in the absence of cilia. Dev Biol 330: 452-460, 2009.

83. Zeng H, Jia J and Liu A: Coordinated translocation of mammalian Gli proteins and suppressor of fused to the primary cilium. PLoS One 5: e15900, 2010

84. Chen MH, Wilson CW, Li YJ, Law KK, Lu CS, Gacayan R, Zhang X, Hui CC and Chuang PT: Cilium-independent regulation of Gli protein function by Sufu in Hedgehog signaling is evolutionarily conserved. Genes Dev 23: 1910-1928, 2009.

85. Ohlmeyer JT and Kalderon D: Hedgehog stimulates maturation of Cubitus interruptus into a labile transcriptional activator. Nature 396: 749-753, 1998.

86. Paces-Fessy M, Boucher D, Petit E, Paute-Briand S and Blanchet-Tournier MF: The negative regulator of Gli, Suppressor of fused (Sufu), interacts with SAP18, Galectin3 and other nuclear proteins. Biochem J 378: 353-362, 2004.

87. Cheng SY and Bishop JM: Suppressor of Fused represses Gli-mediated transcription by recruiting the SAP18-mSin 3 corepressor complex. Proc Natl Acad Sci USA 99: 5442-5447, 2002.

88. Lin C, Yao E, Wang K, Nozawa Y, Shimizu H, Johnson JR, Chen JN, Krogan NJ and Chuang PT: Regulation of Sufu activity by $\mathrm{p} 66 \mathrm{~b}$ and Mycbp provides new insight into vertebrate Hedgehog signaling. Genes Dev 28: 2547-2563, 2014.

89. Barnfield PC, Zhang X, Thanabalasingham V, Yoshida M and Hui CC: Negative regulation of Gli1 and Gli2 activator function by Suppressor of fused through multiple mechanisms. Differentiation 73: 397-405, 2005.

90. Kogerman P, Grimm T, Kogerman L, Krause D, Undén AB, Sandstedt B, Toftgård R and Zaphiropoulos PG: Mammalian suppressor-of-fused modulates nuclear-cytoplasmic shuttling of Gli-1. Nat Cell Biol 1: 312-319, 1999.

91. Ruiz i Altaba A, Sánchez P and Dahmane N: Gli and hedgehog in cancer: Tumours, embryos and stem cells. Nat Rev Cancer 2: 361-372, 2002.

92. Humke EW, Dorn KV, Milenkovic L, Scott MP and Rohatgi R: The output of Hedgehog signaling is controlled by the dynamic association between Suppressor of Fused and the Gli proteins. Genes Dev 24: 670-682,2010.

93. Merchant M, Vajdos FF, Ultsch M, Maun HR, Wendt U, Cannon J, Desmarais W, Lazarus RA, de Vos AM and de Sauvage FJ: Suppressor of fused regulates Gli activity through a dual binding mechanism. Mol Cell Biol 24: 8627-8641, 2004.

94. Dunaeva M, Michelson P, Kogerman P and Toftgard R: Characterization of the physical interaction of Gli proteins with SUFU proteins. J Biol Chem 278: 5116-5122, 2003.
95. Chen Y, Yue S, Xie L, Pu XH, Jin T and Cheng SY: Dual Phosphorylation of suppressor of fused (Sufu) by PKA and GSK3beta regulates its stability and localization in the primary cilium. J Biol Chem 286: 13502-13511, 2011.

96. Wang Y, Li Y, Hu G, Huang X, Rao H, Xiong X, Luo Z, Lu Q and Luo S: Nek2A phosphorylates and stabilizes SuFu: A new strategy of Gli2/Hedgehog signaling regulatory mechanism. Cell Signal 28: 1304-1313, 2016.

97. Zhou F, Huang D, Li Y, Hu G, Rao H, Lu Q, Luo S and Wang Y: Nek2A/SuFu feedback loop regulates Gli-mediated Hedgehog signaling pathway. Int J Oncol 50: 373-380, 2017.

98. Liu C, Zhou Z, Yao X, Chen P, Sun M, Su M, Chang C, Yan J, Jiang $J$ and Zhang Q: Hedgehog signaling downregulates suppressor of fused through the HIB/SPOP-Crn axis in Drosophila. Cell Res 24: 595-609, 2014.

99. Lee DY, Deng Z, Wang CH and Yang BB: MicroRNA-378 promotes cell survival, tumor growth, and angiogenesis by targeting $\mathrm{SuFu}$ and Fus-1 expression. Proc Natl Acad Sci USA 104: 20350-20355, 2007.

100. Long H, Wang Z, Chen J, Xiang T, Li Q, Diao X and Zhu B: microRNA-214 promotes epithelial-mesenchymal transition and metastasis in lung adenocarcinoma by targeting the suppressor-of-fused protein (Sufu). Oncotarget 6: 38705-38718, 2015.

101. Peng RQ, Wan HY, Li HF, Liu M, Li X and Tang H: MicroRNA-214 suppresses growth and invasiveness of cervical cancer cells by targeting UDP-N-acetyl-a-D-galactosamine:pol ypeptide $\mathrm{N}$-acetylgalactosaminyltransferase 7. J Biol Chem 287: 14301-14309, 2012.

102. Browne G, Dragon JA, Hong D, Messier TL, Gordon JA, Farina NH, Boyd JR, VanOudenhove JJ, Perez AW, Zaidi SK, et al: MicroRNA-378-mediated suppression of Runx1 alleviates the aggressive phenotype of triple-negative MDA-MB-231 human breast cancer cells. Tumour Biol 37: 8825-8839, 2016.

103. Min TH, Kriebel M, Hou S and Pera EM: The dual regulator Sufu integrates Hedgehog and Wnt signals in the early Xenopus embryo. Dev Biol 358: 262-276, 2011.

104. Peng Y, Zhang X, Ma Q, Yan R, Qin Y, Zhao Y, Cheng Y, Yang M, Wang Q, Feng X, et al: MiRNA-194 activates the Wnt/ $\beta$-catenin signaling pathway in gastric cancer by targeting the negative Wnt regulator, SUFU. Cancer Lett 385: 117-127, 2017.

105. Bari R, Hartford C, Chan WK, Vong Q, Li Y, Gan K, Zhou Y, Cheng C, Kang G, Shurtleff S, et al: Genome-wide single-nucleotide polymorphism analysis revealed SUFU suppression of acute graft-versus-host disease through downregulation of HLA-DR expression in recipient dendritic cells. Sci Rep 5: $11098,2015$.

106. D'Amico D, Antonucci L, Di Magno L, Coni S, Sdruscia G, Macone A, Miele E, Infante P, Di Marcotullio L, De Smaele E, et al: Non-canonical Hedgehog/AMPK-mediated control of polyamine metabolism supports neuronal and medulloblastoma cell growth. Dev Cell 35: 21-35, 2015. 